\title{
التعددية اللغوية فى القارة الإفريقية \\ وأثرها على أدب الطقل
}

\author{
د. ربيع فتحي علي محمد الحسيني
}

دكتوراه في فلسفة الإدارة العامة ـ جامعة بني سويف لفيف

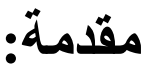

إفريقيا أصل الحضارة الإنسانية ومنبعها، فمنها خرج الإنسان الأول و على أرضها نشأت أقدم الحضار ات.

و العولمة إذا كانت تشكل تحديًا لقارتنا الإفريقية، إلا أنه يجب أن نجعل منها فرصة جديدة تتيح لثقافتنا في القارة الإفريقية المزيد من النمو والنضج و الإسهام في رقي الإنسانية.

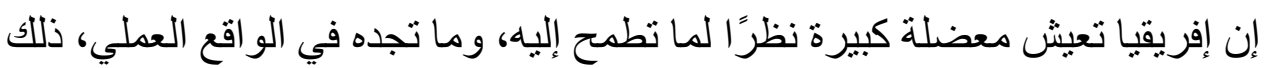
أنها كلما خطّطت لإجر اء إصلاحِ تربوي ثقافي أدبي داعِ إلى تطوير لغاتهم و آدابهم الأصلية اصطدمت بتنمية لغاتٍ غربية مقابل إعفاء الديون أو التخفيف منها.

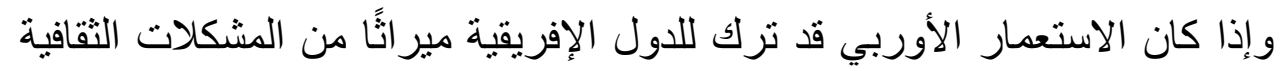
و الاجتماعية والاقتصادية، إلا أن قدرة مثققي القارة على تجاوز ها باتت فى هذه الآونة أقوى من أى زمنٍ مضى. إن معركة الثقافات هي السمة البارزة في المشهد الثقافي الإفريقي؛ نظرًا لوجود بقايا

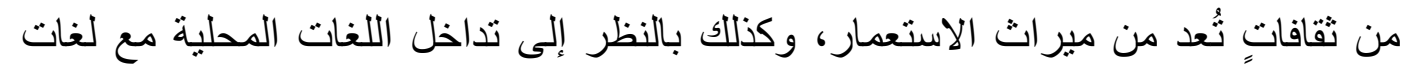
المستعمر السابق ومنها: الإنجليزية و الفرنسية والإسبانية والبرتغالية، معتبرًا ذللك إحدى الشكلات التي تواجه أدب الطفل الإفريقى، ليس على مستوى القارة الإفريقية (بين دولة

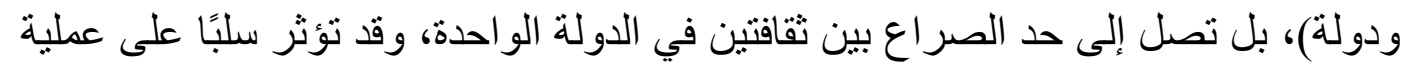
بناء الثخصية الأدبية داخل الدولة الواحدة.

\section{القارة الإفريقية ومشكلة التواصل المعرفى:}

وفى الوقت الذي يرى فيه المراقبون أن مستقبل العالم مرتبطُ بالقارة الإفريقية مما

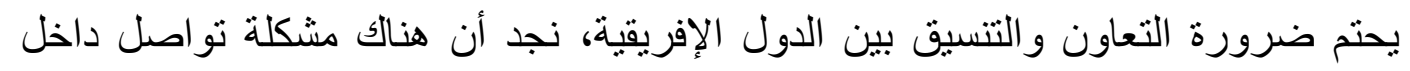


القارة الإفريقية، وهو ما يجعل الفجوات المعرفية والثقافية بين الدول الإفريقية المختلفة كبيرة. فإفريقيا التي هى ثانى أقدم قار ات العالم من حيث المساحة و السكان وتتكون من ( ع) دولة، ترجع مشكلاتها التي خلّفتها الحدود المرسومة بين الدول الإفريقية إلى الطريقة التي اتبعها الأوربيون فى تقسيم إفريقيا فيما بينهم أثتاء مؤتمر برلين 110 (، حيث لم تراع الدول الأوربية أي اعتبارات خاصة بالأفارقة، مثل: التجانس العرقى واللغوى، و التكامل الاقتصادى، والإرث الحضارى، كما أن الدول الإفريقية لم تُعط أى فرصة لإبداء رأيها، و إنما لعبت المنافسة والتسابق والاستحو اذ على مناطق النفوذ دورًا مهمًا فى وضع الحدود. إلا أننا نستطيع أن نجعل من الثقافة منطقة لتفاعل الأفارقة جميعًا، فكيف يمكنتا ذلك؟

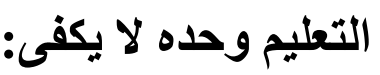

إذا كان التعليم هو اللبنة الأولى في بناء شخصية الإنسان وصقلها ووضعها على الطريق الصحيح لتخريج أجيالاً متمكنة من العمل والمساهمة في عملية التنمية المنشودة في الدولة، فإن ذلك يبقى من دون قيمة وبلا أهمية ما لم تُحدد معالم وملامح الهوية الوطنية، ودعمها وتعزيز ها ونرسيخها في نفوس و عقول وقلوب أبناء الوطن، أجيال الحاضر ورجال المستقبل، الأمر الذي يؤكد أهمية وضرورة الثقافة، ودور ها المحوري في تحقيق ذلك، لتشكل مع التعليم جناحي الدعم و التعزيز للهوية الوطنية للقارة و التي ننشدها جميعًا. و على الرغم من أهمية التعليم في بناء وتعزيز الهوية الوطنية، لكن لا يجب أن

نحمّل وزارات التربية والتعليم المسئولية كلها، بل تتحمل ذلك كل المؤسسات بما فيها الإعلامية و التعليمية و التربوية، وبر امج التليفزيون والإنترنت وغير ها من المكونات التي تبني ثقافة الإنسان وتؤثر فيه، وبالتالي فإن المدرسة وحدها وفي ظل هذه الأجواءالتي تمر بها القارة لن تستطيع بناء هوية الطفل، حتى لو تم تطبيق أحدث المناهج وبأفضل المعلمين،

$$
\text { فإنها لن تجدي نفعًا؛ لأن الأساس في العملية هو التربية. }
$$

إن تعدّد الثقافات في الدولة والجنسيات ذات العادات والتقاليد المختلفة بالضرورة أن

تؤثر تللك العادات سلبًا على هويتهم الوطنية، و المجتمع الذي لا هوية موحدة له لا وجود له. 


\section{المشهد اللغوي في إفريقيا .. المسار والعوائق:}

في الواقع، هناك أنشياء يجب إزالة الضباب عنها، منها أصالة اللغات الإفريقية

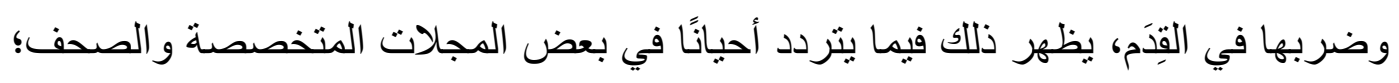

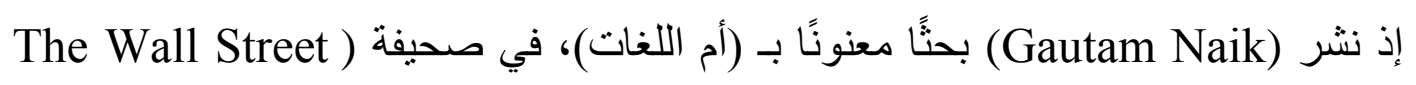

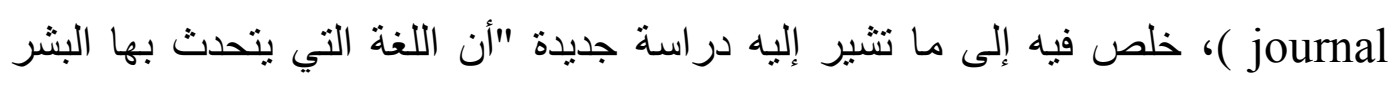

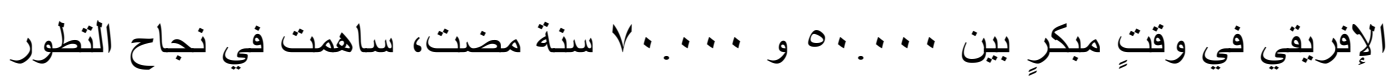

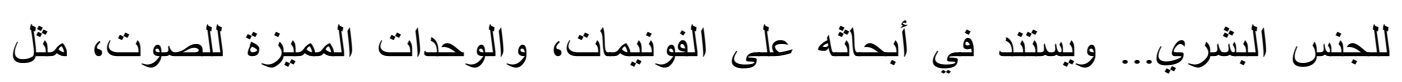
حروف العلّة، ونغمات الحروف الساكنة".

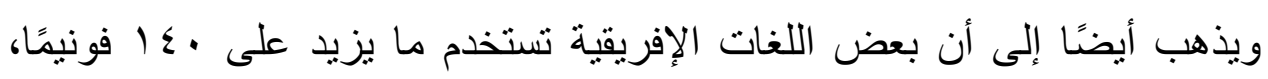
مقارنًا ذللك بمثيلاتها في قار اتٍ أخرى تستعمل أقل من ذلك كثيرًا، فقارنها بغير ها ليستتنج أنها ساهدت في تطوير الفونيم أساسًا، ثم تأثير ها في غير ها من اللغات العالمية المختلفة.

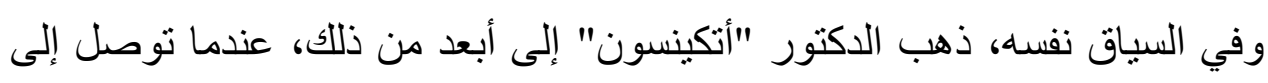

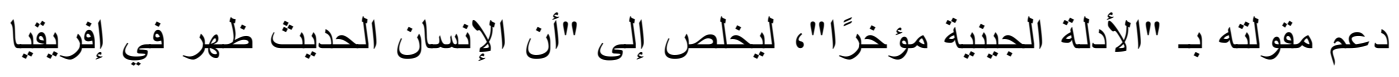
وحدها".

وفي السياق نفسه، نشر(The roo) مقالة في صحيفة (1n McWhorterJoh) بعنوان (للتعرف على اللغات؛ اسأل إفريقيا تخبرنا عن أصولنا)، استند المؤلف إلى أدلة

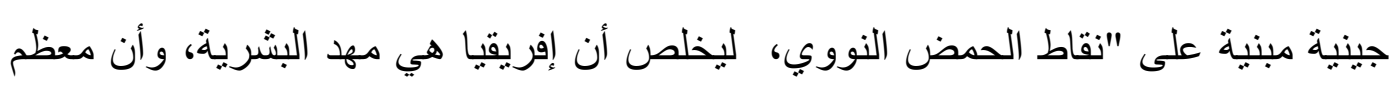
أصوات المعمورة تنحدر منها".

إن الذي يتتبع الظروف التي تمر بها اللغات الإفريقية لا ينتابه شك في أنها تعيش

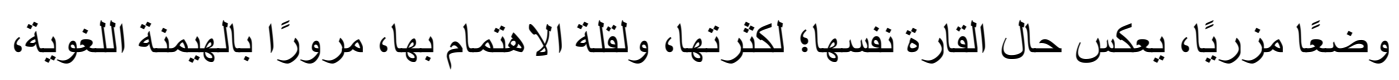

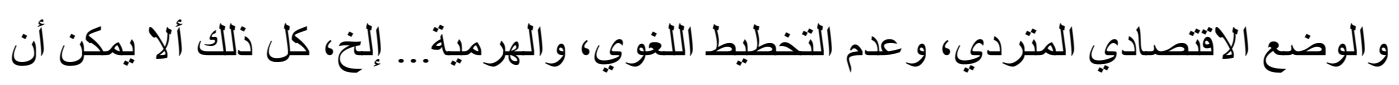

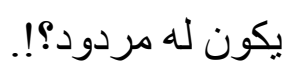

وعليه، يمكن أن نطرح السؤال الآتي: ما الظروف التي تمر بها اللغات الإفريقية؟ وما العو ائق التي تحول دون تطور هاب ان نطرح 


\section{أولاً- وضع اللغات الإفريقية:}

إن لغات العالم بفصائلها وفرو عها لم تعد تُحصى بالعشر ات ولا بالمئات، بل أضحت تُعد اليوم بالآلاف؛ إذ إن عددها في حياة نشطة وتز ايد مستمر، من جانب آخر يُظهر التوزيع الجغر افي للغات العالم مشكلة من حيث تفاوت كثافتها الذي بتمثل في وجود ثلثيها تقريبًا "في (9) دول فقط". (9)

إذا كان ثلث لغات العالم توجد في القارة السمراء، فإن ثمة مشكلة تظهر من حيث التوزيع الجغر افي لهذه اللغات؛ إذ توجد نصف لغاتها تقريبًا في ثلاث دول فقط، كما مرّ أعلاه، فكان من المنطق أن تستعمل كل لغة أبجديتها الخاصة، والتي تعبر عن نظامها الصوتي و الصرفي والنحوي والتركيبي... إلخ، ولكن ما حدث عكس ذللك، فإن عدد الأبجديات حتى الآن عالميًا في حدود المائة، و على ضوء ذلك يمكن استتناج أن أغلب اللغات

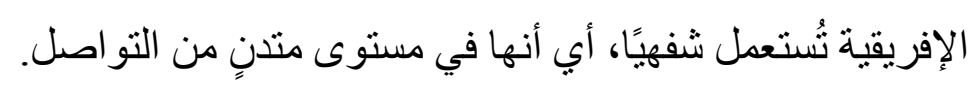
و إذ كانت اللغات الإفريقية بهذا العدد العظيم، فإن الثعوب التي جنحت إلى وضع أبجديتها الخاصة أو ترسيم أبجدية لاتينية لنفسها قليلة في القارة السمراء، و عليه يمكن استنتاج أن مئات اللغات الإفريقية في خطرٍ محدق؛ لكونها لا تُشتعمل في مستوى عالٍ من التخاطب، و إنما يقتصر استعمالها على شئون الحياة اليومية، وقد أثبتت بعض البحوث أن ثمة لغة تموت عالميًا في متوسط كل أسبوعين، ويهدد هذا الموت أغلب اللغات الإفريقية، و التي لم يفعل أهلها الثيء الكثير لحماية تر اثهم اللغوي الذي يضمحل يومًا بعد آخر. وفي الو اقع، هناك محاو لات فردية ومجهودات مشنتة منذ عقود - إن لم نقل قرونلوضع الأبجديات في مختلف لغات إفريقيا، ويلاحظ أن بعضها تأثر بالعربية أو اللاتينية، بيد أن هنالك أبجديات أخرى يمكن وصفها بالمحايدة، إلا أن القليل منها يحظى بالعيش إلى عقدٍ أو عقودٍ أو قرون؛ ذلك أن بعضها يموت بموت المؤلف؛ إذ إن حيوية هذه الأبجديات ودعمها يعتمدان على الجماعة اللغوية، و التي لا تستسيغ هذا الدعم ثقافيًا، أضف إلى ذلك عدم اعتبار الحكومات و المنظمات غير الحكومية لها؛ ذلك أن أغلب الحكومات تعتمد أبجديات لاتينية، بناءً على هيكلة اللغات الرسمية، فتسعى إلى خلْق انسجام بين مختلف اللغات الوطنية، وذللك بفرض أبجدية ثابتة عليها، وهي غالبًا ما تكون لاتينية. 
وعلاوة على ذللك، فإن أغلب الثعوب التي عرفت الثقافة الإسلامية في إفريقيا ـ منذ قرون- قامت بوضع أبجديات عربية، أو متأثرة بها للغاتهم، واستعملوها في كتابة نتاجهم؛ لتترك تراثًا علميًا غزيرًا في مختلف مجالات العلم، والذي يزور مر اكز المخطوطات في مختلف عو اصم الثقافة الإفريقية ينتابه العجب من غز ارة هذه المؤلفات ومحتو اها العلمي. ولار اسة الموضوع من بابه الواسع، فإن اللغة تتعلق بالهوية، وما فقته القارة من هويتها لا يقل عما فقدته من لغاتها، فهي تعيش صر اعًا كبيرًا، ذلك أن أغلب شعوب القارة تأثرو الالثقافة الغربية، وبخاصة المتغربون الذين نهلو المنها وأُشربو ا حبها، فأضحو ا يعيشون اليوم حائرين! بل تاهو ا، وآن الأوان أن ينظروا مجددًا إلى مجدهم ليفهموا "أن الهوية تُنقش بواسطة اللغة والثقافة والتاريخ، ويتوصل إلى كل ذلك عبر التعليم، في إفريقيا تختنق الهوية بثقل كاهل الاستعمار الثقيل الذي أنشأ البُنى المحلية لترسيخ إدارته، وفرض نظامًا تعليميًا، بما في ذلك اللغات و التقافات و التواريخ التابعة للدول الغربية". إن بعض الدول الإفريقية تفتح أبو ابها على مصر اعيها لاستيعاب كل أنواع الثقافات المختلفة، حتى أضحى بعضها بذلك سوقًا استهلاكية لكل غتّ أو سمين منها، وظل بعضها أسيرًا أيضًا للنفوذ اللغوي الإمبريالي القائم أحيانًا على ما يُسمى (العلاقة الثقافية)، فتسعى إلى عقد صفقاتٍ بأي ثمن لاستعمال لغاتها في مستوى معين من الدراسة، كل ذلك قصد الحصول على أصدقاء جدد في الأسـواق الإفريقية، فهذه المنظمة الفرنكوفونية "تترقب في عام •0. • ام أن تصل إلى 0^\% من مستعملي اللغة الفرنسية في إفريقيا وحدها". و على مستوى التغطية الإعلامية بين لغات العالم، يُلاحَظ أن هنالك وضعًا ينذر بالخطر؛ لأن • ^^\% من الإعلام العالمي ينحصر في بی لغة، في موضوعاتٍ حاسمة على الشبكة العنكبوتية، وأغلب المحتويات (التي تصل إلى ،9\%) توجد في با ب\% من لغات العالم، و عليه فهناك هوة كبيرة بين مختلف اللغات. وقد قام خبراء في اللسانيات باليونسكو بجمع معلومات حول . .0 لغة، ووضع

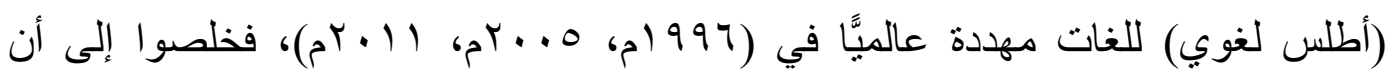
إفريقيا تعاني مشكلة وباء (الصراع اللغوي) فمثلاً: (نيجيريا، الكاميرون، ج. كونغو الديمقر اطية، تنز انيا، السودان، إثيوبيا، تشاد) تدخل في عِداد مجموعة الـ هب دولة التي 
ومع ذللك، فإن اللغات الإفريقية بالر غم مما تشهده من تفاوتٍ في الاستعمال و التعليم، من حيث الإقبال الكبير لدى جماعة، و المتوسط لدى أخرى، فإنها تُستعمل في وسائل الإعلام

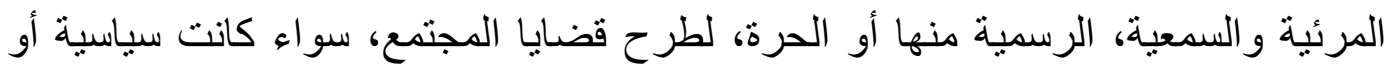

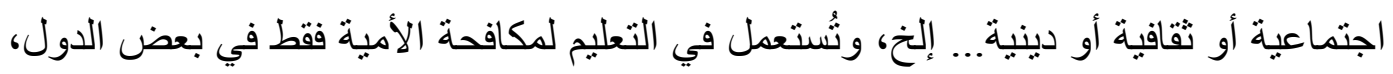
أو في المرحلة الأساسية في دول أخرى، ولدى بعض آخر إلى إلى المرحلة الثانوية، كما أنها تُدرس إلى مستوى الجامعة في دول أخرى من القارة السمر اءهاء.

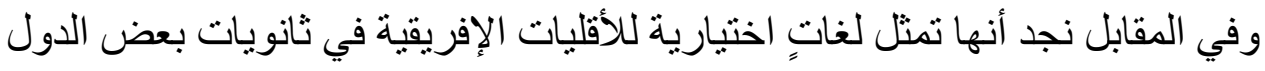

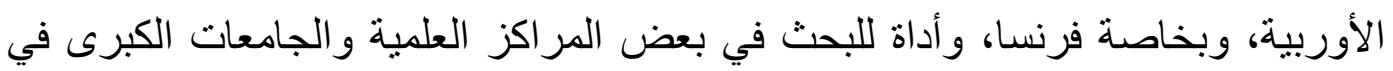
أوربا وأمريكا، وفي طليعة هذه اللغات: اللغات الحدودية، أو الغالبة، وذهبت الأكاديمية ولهية

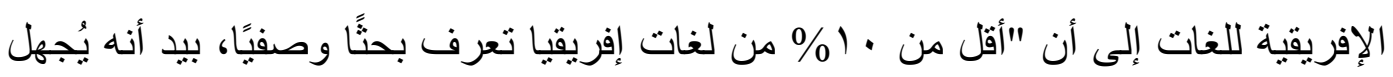

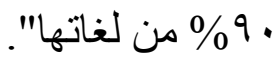

\section{ثانيًا - عوائق تظور اللفات الإفريقية:}

إن اللغات الإنسانية تتطور بمجموعة من الوسائل و الآليات، وتتعرض أحيانًا للخطر

$$
\text { و المشكلات، والتي تعود إلى عوامل داخلية أو خارجية. }
$$

و على ضوء ذلك يمكن إير اد بعض العو امل التي حالت دون تطور اللغات الإفريقية:

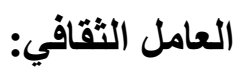

إن القارة الإفريقية غنية بتعدد ثقافاتها وحضار اتها؛ إذ نشأت فيها العديد من الممالك

$$
\text { و الإمبر اطوريات في الثرق و الغرب. }
$$

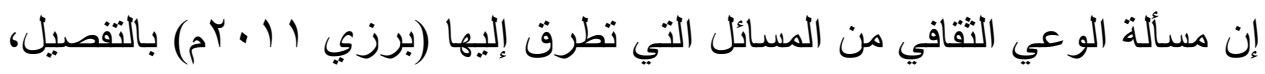

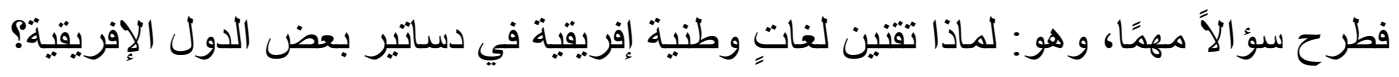

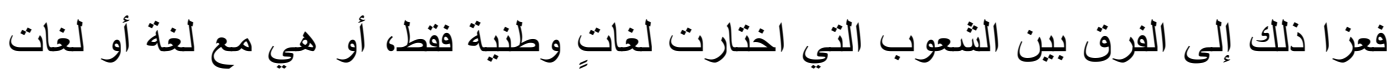
غربية في ترسيم مسيرتها اللغوية، و التي اختارت لغة أو لغات استعمارية فقط في سياساتها

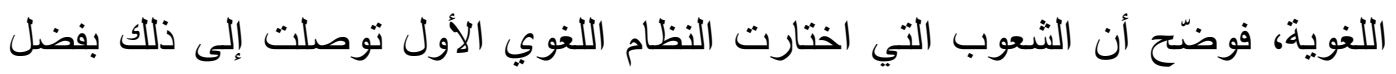
و عيها الثقافي المو غِل في القِدَم. وعليه، فان إيلاء أية أهمية للنقافة يؤدي بالطبع إلى تنمية لغوية قومية، وعكسه يؤدي إلى إخفاق اللغات، وربما إلى موتها أحيانًا. 
وأعتقد أن ما طرحه (ويدر اغو) من أن "صعوبة الانتقال تُعد عائقًا" يمثل جزءًا من

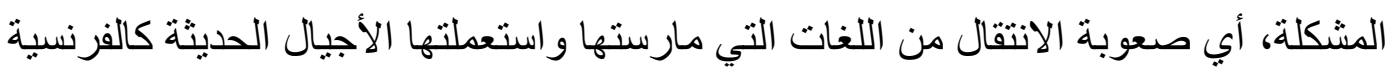

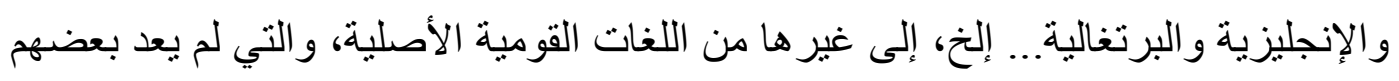

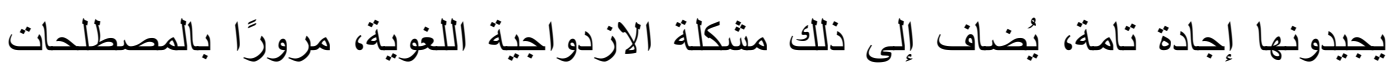
التقنية و العلوم التي حظيت بها اللغات الاستعمارية. كما أن طرحه صحيح - حسب اعتقادي - من ناحية أخرى؛ لأن ما ترسّخ في مفهوم

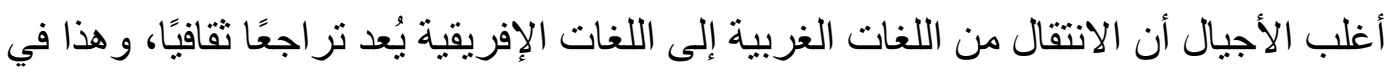
الو اقع مشكلة خطيرة من مشكلات الغزو الفكري، قد تجعل الباحث يتثاءم بشأن أي إصلاح لغوي في السنوات القادمة، حينما لا توجد قناعة وثقة لاى مستوى معين مين من النخبة المثنقة من الثعب بلغاتهم الأصلية، ومع ذللك يُلاحظ أن المشكلة متفاونة بين الدول الإفريقية.

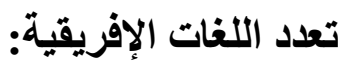

إن شعوب القارة الإفريقية غارقة في تعدد لغوي خطير، ومن ثم فإن انتخاب اللغة

التعليمية أو الرسمية ليس بالهيّن ما دامت كل لغةٍ تسعى إلى الهيمنة.

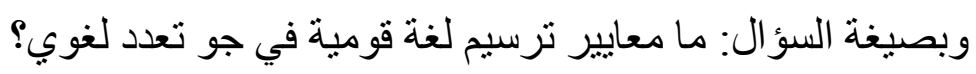

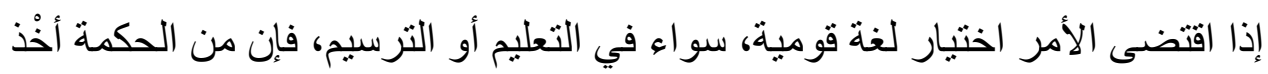

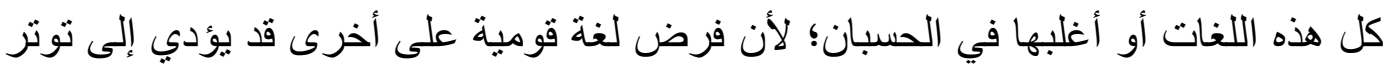

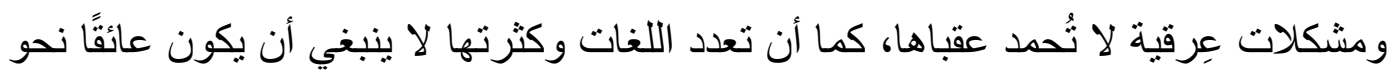

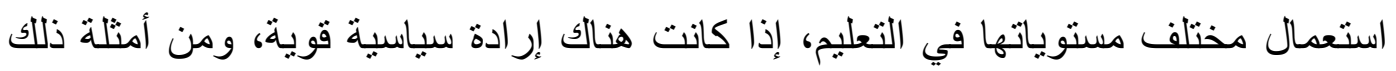
(بابوزي) التي "اسنطاعت في ثلاث سنو ات فقط وضع نظام تعليمي لأكثر من نصف لغاتها

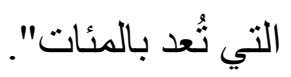

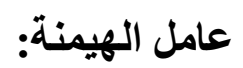

من المسلَّمات أن النظام الإمبريالي أيضًا له يدٌ طولى في عرقلة تطور اللغات

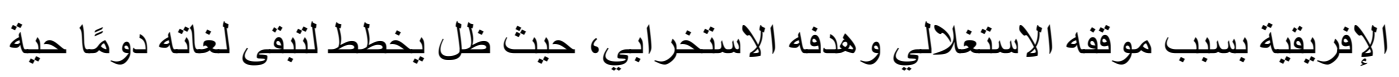

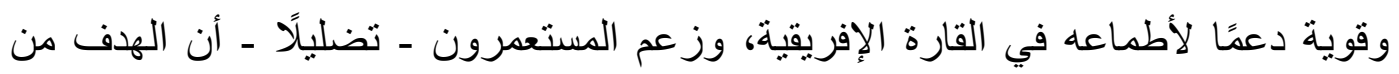
تغريب الأفارقة، بما في ذلك تحريضهم على استعمال لغات غربية، هو "تمدين الإفريقي 
الأصلي، وكأن أبي إفريقي منمدن هو الذي بإككانه وحده أن يأمل في الحصول على وظيفة

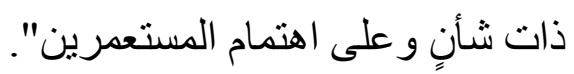

هذا بالإضافة إلى موقف الغرب المتناقض حول قيمة الثقافة الإفريقية، مرورًا

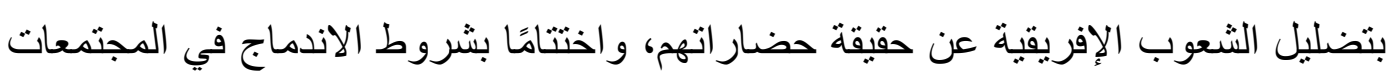

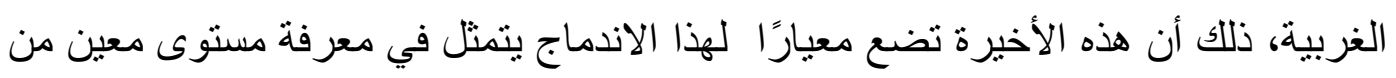

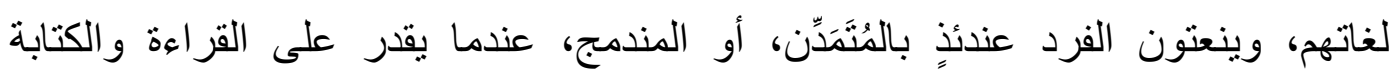

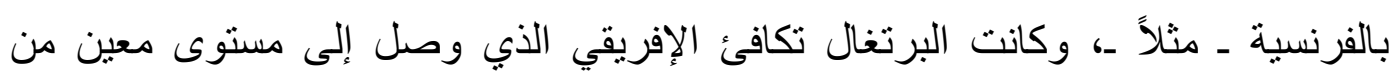

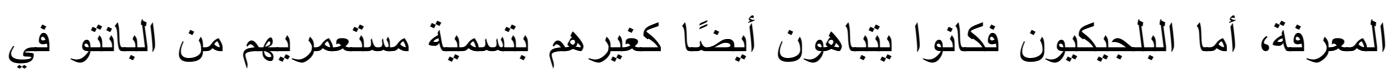

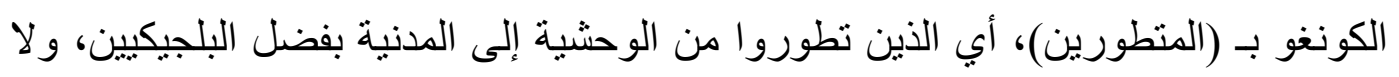

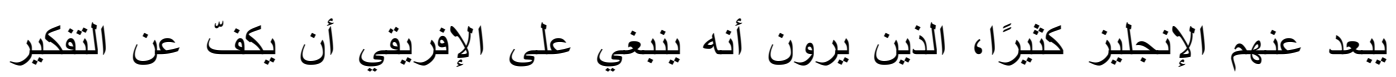

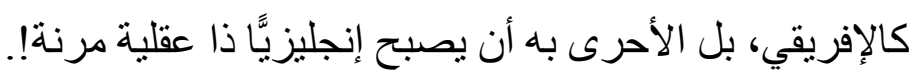

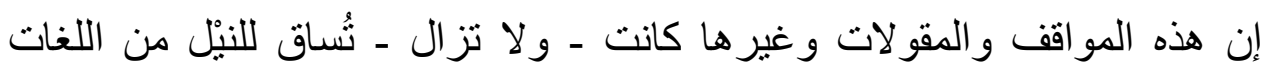

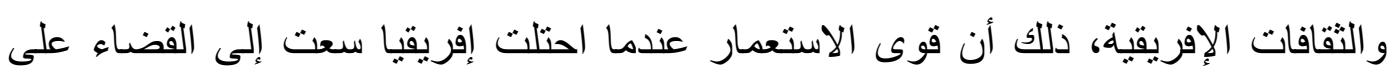
اللغات الأصلية بحجة أنها لا اعتبار لهاو لا تصلح في تنمية شعوبها.

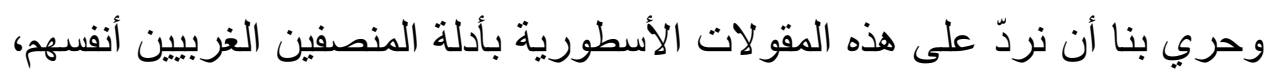

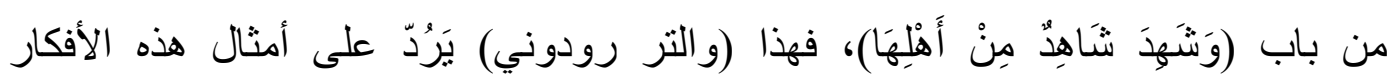

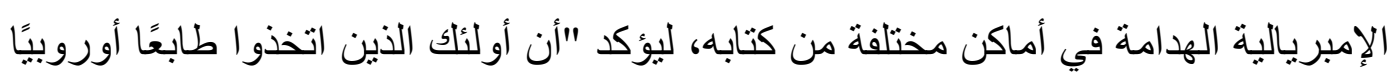

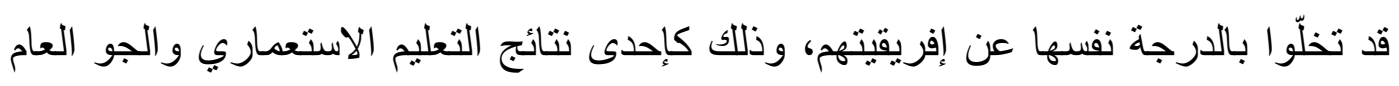
للحياة الاستعمارية". ولا ننسى - مبدئًًا ـ أن الدول الكبرى لا تقبل لمستعمر اتها أبدًا المبادرة بتنمية لغاتها

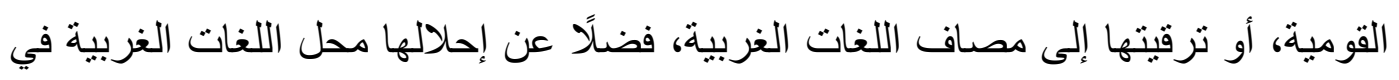

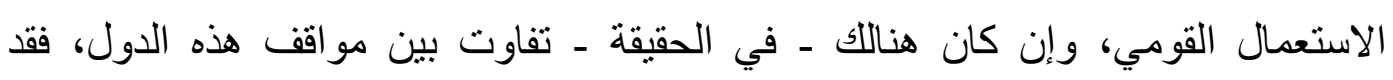

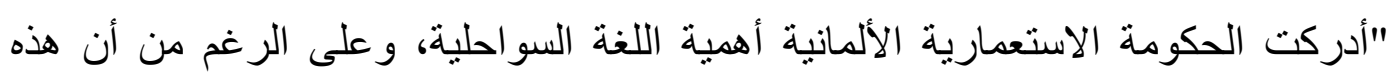

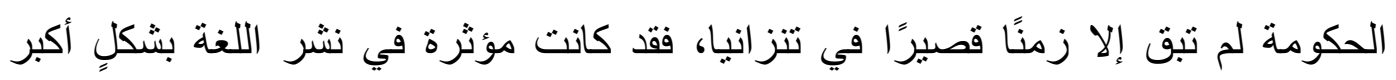

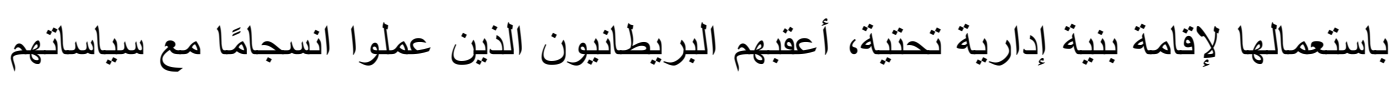

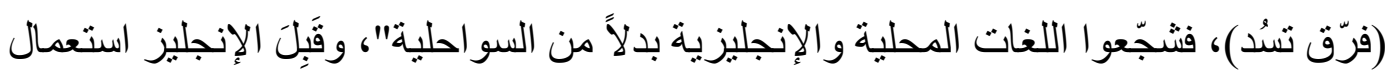


اللغات الأصلية في التعليم الذي يُعد نظامًا شبه منتشر في مختلف المستعمرات، وبخاصة

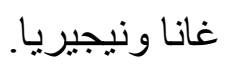

وفي المقابل، فإن فرنسا تعارض ذللك بكل ثمن، فلا تتوانى في خلْق أفظع أنواع

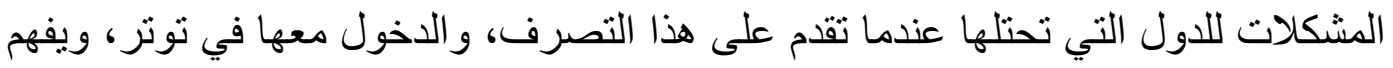

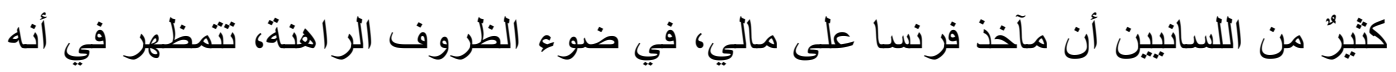

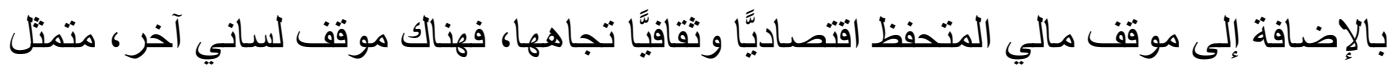

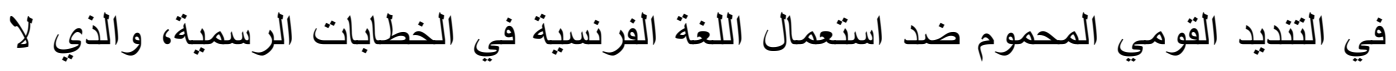
يمكن أن يناقش حسب علمي في مستويات رفيعة بصورة سهلة في كثيرٍ من الدول الإفريقية الفرنكوفونية، حيث دعت الحركات القومية إلى إحلال اللغات الوطنية مقام اللغة بنة

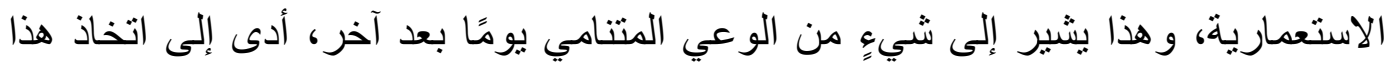

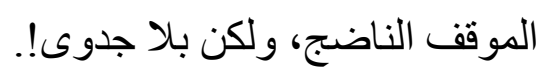

ولا تبعد عنها "الجابون" التي أضحت تستعمل اللغة الإنجليزية في نظامها التعليمي

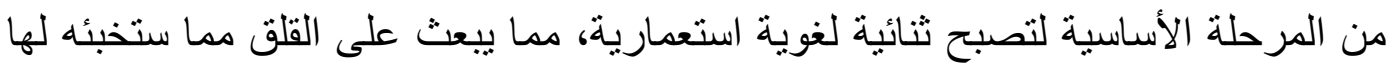
الأيام القادمة!.

ويؤكد (ويدر اغو) الفكرة نفسها في أماكن كثيرة من كتابه، وبصورة واضحة، يمكن

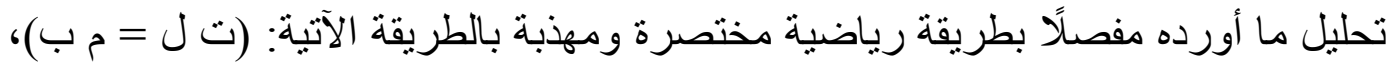
تعني هذه الدائرة أن (التخطيط اللغوي أو التربوي) يساوي (المشي على البيض) في إفريقيا،

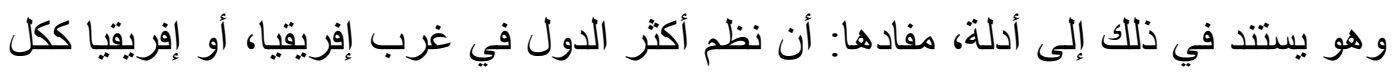

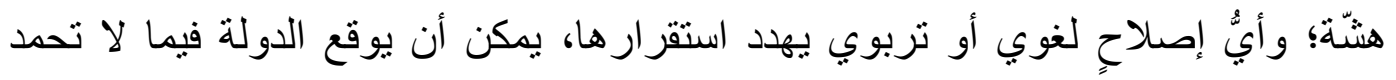

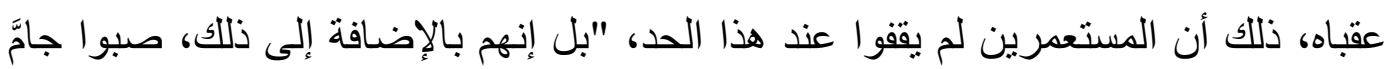
عنصريتهم على الأفارقة منلما فعل هتلر مع اليهود". عامل التخطيط اللغوي: إن التخطيط عاملّ مهُم في تأسيس الدول، وهذا التخطيط، سواء أكان قريبًا أو بعيد

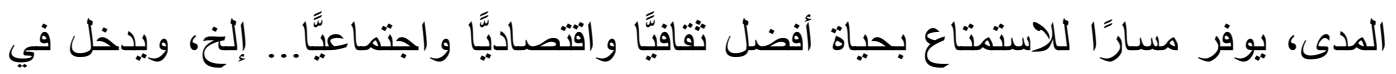
ذلك "التنبؤ باحتباجات المستقبل في ضوء إمكانات الحاضر والقوة المؤثرة فيه، والعمل الإيجابي الهادف نحو تحقيق هذه الاحتياجات". 


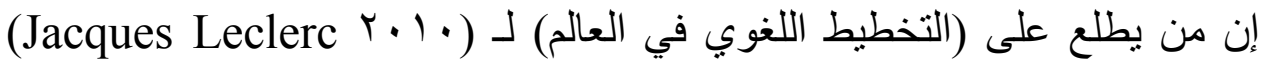
و الذي يدور حول لغات إفريقيا، واحدة تلو الأخرى، وطريقة ترسيمها وتخطبطها، سواء

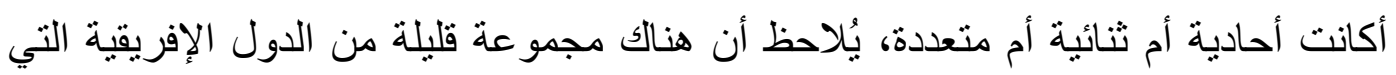
اختارت أحادية لغوية وطنية في قانونها الأساسي، لتكون صدى لسياستها اللغوية.

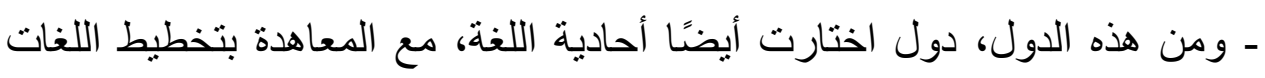
الوطنية. - ومن هذه الدول، دول اختارت ثنائية لغوية مؤسسية، فساوت بين اللغات

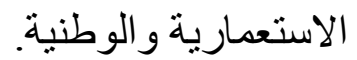
ـ ـُيلاحَظ أن هناك دو لاًا اختارت ثنائية لغوية استعمارية رسمية.

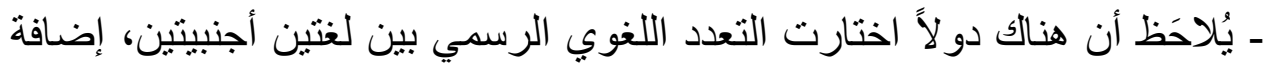
إلى لغاتٍ وطنية. - في المقابل، هناك دول انتخبت لغات استعمارية فقط في قو انينها، أي لم تولِ أيَّة أهمية للغاتها الأصلية. إن التربية والتعليم تتطلبان مجهودًا على مستوى التجهيز، أو على مستوى التقويم و التخطيط، كل ذلك لا يمكن أن يتحقق إلا بالقدرة الاقتصادية، وفي الواقع تحسّن (التربية المتقاربة) CONVERGENTE PEDAGOGIE من مردودات التعليم الأساسي؛ وقد أثبتت النتائج أن عدد المتعلمين في تقدٍٍ اليوم، ومع ذلك فإن تكاليف (التربية المتقاربة) لكل

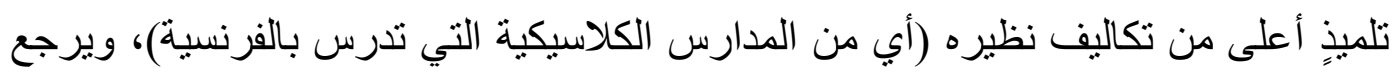
هذا في جانب منه إلى تكاليف الاستثمار في مرحلة التوسع المرتبطة بتكوين المعلمين. ونظرًا للعجز الاقتصادي الذي تعيش فيه أغلب الدول الإفريقية، فإن ميزانية التعليم

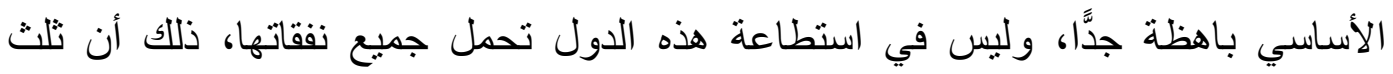

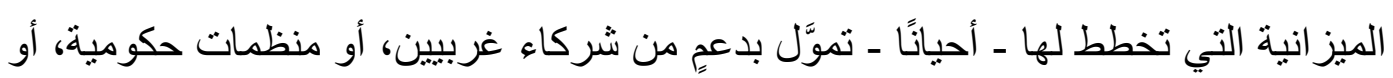

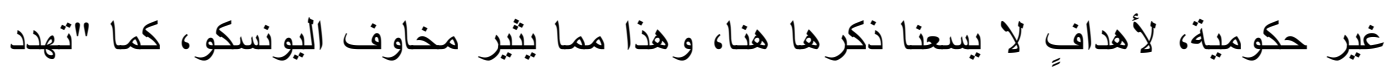

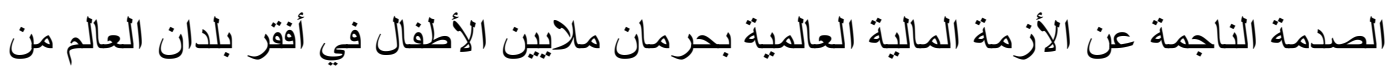

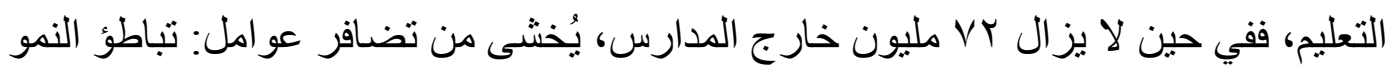


الاقتصادي، نز ايد الفقر، وضغوط الميزانية، أن يقوض المكاسب المحصّلة في ميدان التعليم خلال العقد المنصرم".

\section{الأدب الأفريقى بين المأثورات الشعبية وأدب الطقل:}

بإنتهاء الحرب العالمية الثانية، ومع تغيُر خريطة السياسة الدولية واشتعال فتنيل الحرب الباردة التي بدت كحلبة صراع للقوى العظمى متمثلة في لاعبين أساسيين، هما:

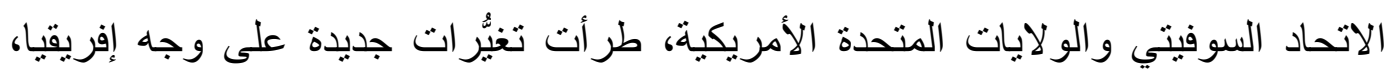

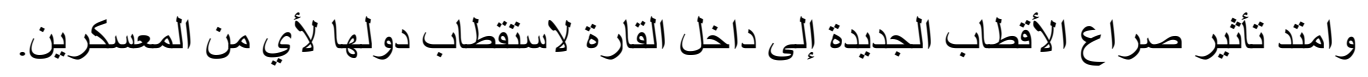

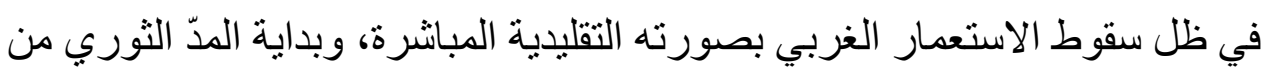

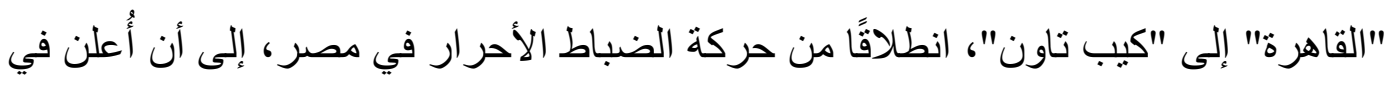
عام •197 وحده عن استقلال 11 1 دولة إفريقية، الأمر الذي استرعى أن يطلق عليه

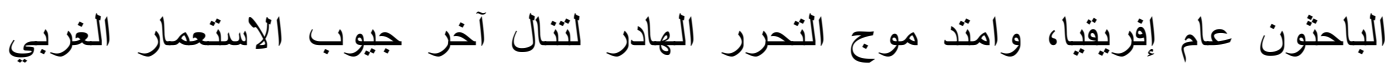
استقلالها في ناميبيا عام • 99 1، بالتز امن مع بداية تحلل نظام الفصل العنصري ـ أبارتهيد -

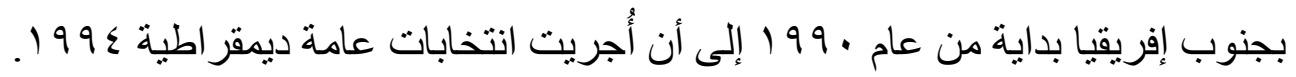

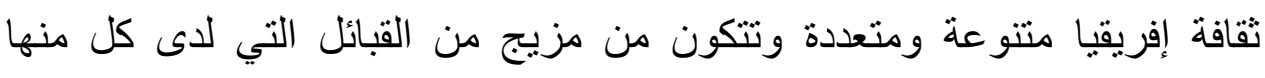

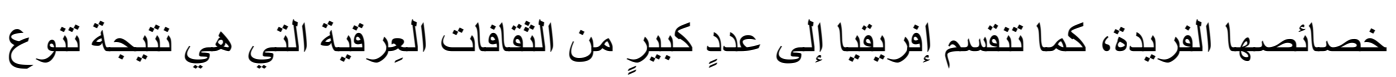

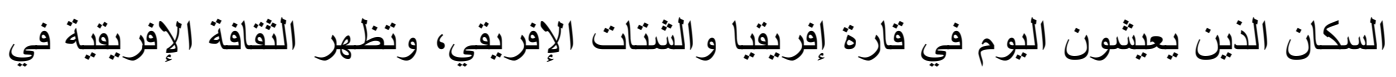

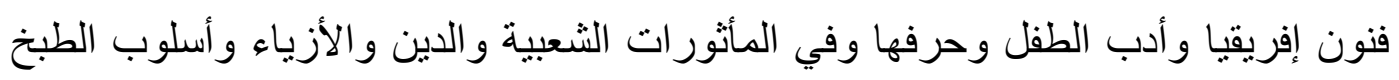

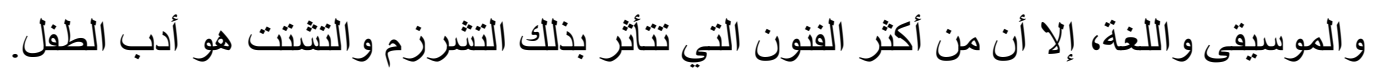

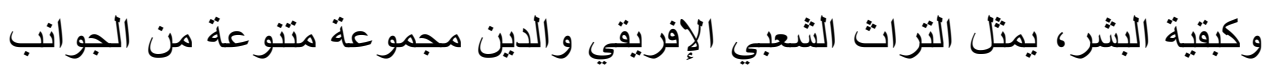
الاجتماعية للنقافات المختلفة في إفريقيا، فتقرييًا كمعظم الحضارات و الثقافات نم تداول

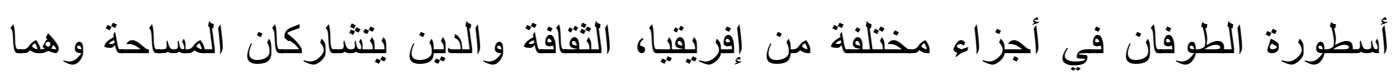
متداخلان بعمقٍ في الثقافات الإفريقية. وفي إثيو بيا مثلًا، المسيحية والإسلام من الجو انب الأساسية للثقافة الإثيوبية و العادات

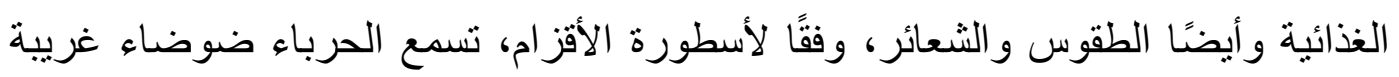
من الثجرة، تقطع جذعها فتخرج المياه متدفقة بشكلٍ قوي ينتشر في جميع أنحاء العالم. 


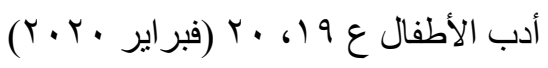
التعددية اللغوية في القارة الإفريقية

تلعب القصص الثعبية دورًا مهمًا في الثقافات الإفريقية، وتعكس القصص هوية جماعة ثقافية والحفاظ على القصص الإفريقية سيساعد على الحفاظ على ثقافة كاملة. وكما أن القصص تؤكد الاعتزاز و الهوية في التقافة، نجد في إفريقيا نؤلف جماعة عِرقية القصص لأنفسهم، مجموعات عِرقية مختلفة في إفريقيا تمارس طقوسًا مختلفة أو احتفالاتٍ للقصص، مما ينمي الشعور بالانتماء إلى جماعة ثقافية. وسماع الأجانب لقصص المجموعات العِرقية يقدّم لهم فكرة عن معتقدات المجتمع و آرائه و عاداته، ويسمح للأشخاص داخل المجتمع أن يقوموا بتمييز مجمو عاتهم، ويظهرون رغبات ومخاوف المجمو عة كالحب والزواج و الموت، و اعتبرت القصص أيضًا كأداة للتعليم و الترفيه، حيث يقدون وسيلة للأطفال لفهم المواد والبيئة الاجتماعية، تتضمن كل قصة أخلاقياتٍ لتعليم الناس، مثل أن الخير سينتصر على الثر، وللترفيه يتم تأليف قصص عجيبة لعالمً غير بشري، عادة، الثخصية الرئيسة للقصة تكون حيوانًا ناطقًا أو شخصية إنسان تحدث له أمور غير طبيعية، وعلى الرغم من أن الحكايات الثعبية للترفيه فإنها تشعر بالانتماء و الاعتز از بالمجتمعات في إفريقيا. هنالك عدة أنواع للقصص الإفريقية: حكايات الحيو انات، والحكايات اليومية، حكايات الحيو انات الأكثر ترفيهًا، مع ذلك تتضمن أخلاقياتِ و عِبرًَا، وحكايات الحيوانات تُقسَّم إلى حكايات المحتال، وحكايات الغول. في حكايات الحيو انات حيوانٌ معين قد تكون له ذات الشخصية دائمًا، أو تكون له شخصية مختلفة في كل قصة كي لا يهتم الجمهور بالشخصيات، الأرنب البري دائمًا هو المحتال، ذكي و ماكر، بينما كان الضبع دائمًا يحتال عليه الأرنب البري. الغيلان دائمًا قاسية ووحوش جَثِعة،، و الطيور كانت هي الرسل في كل القصص. الحكايات اليومية هي أكثر الحكايات جدية لا تتضمن فكاهة أبدًا، وهذا بوضح الحياة اليومية و النضالات في المجتمع الإفريقي، هذه الحكايات تتخذ مسائل مثل المجاعة و الهرب من الموت والتودد وشئون الأسرة، باستخدام أسلوب الأغنية عندما كانت تقال نهاية القصة. كانت هذه مقدمة مهمة لفهز السياق التاريخي والسياسي الذي تشكل فيه الأدب الإفريقي الحديث، والذي عبَّر عن آمال القارة المتحررة لتو ها بلغات المستعمرين. 
يقول الكاتب النيجيري الثهير تثينوا أتثيبي: "لا يمكن أن تحشر الأدب الإفريقي

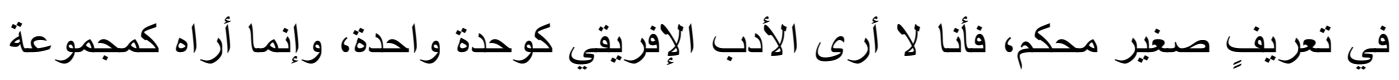

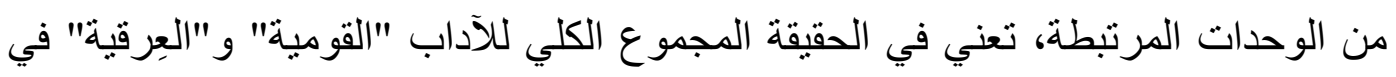
إفريقيا".

فالأدب الإفريقي ككل آداب الأمم مرَّ بمر احل التكوين الأساسية، و التي بدأت بالنقل

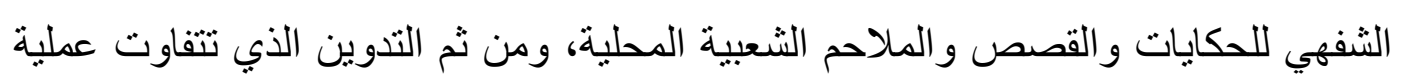

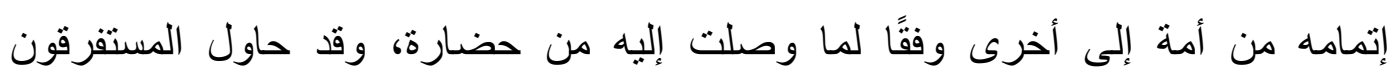

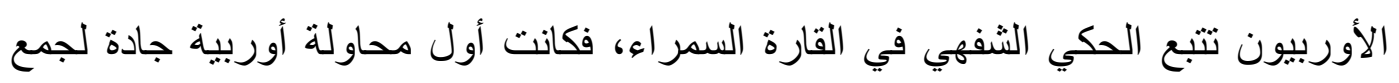

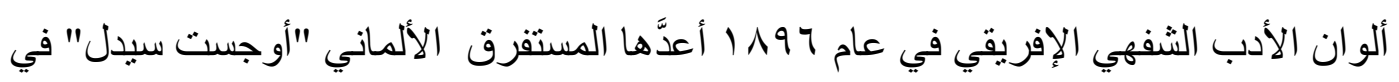
صورة كتاب ضخم تحت عنوان "قصص الإفريقيين وحكاياتهم"، وقد دعا "سيدل" القارئ في تقديمه للكتاب إلى رؤية الإفريقي المتوحش وتخيله وهو يفكر ويشعر ويتخيل وينظم الشعر مثل بقية البشر.

وتو الت محاولات نقل الأدب الثفهي الإفريقي من كلام تلوكه الأفواه إلى كلماتٍ محفوظة في كتب، فجاء المستفرق الألماني "ليوفروبنيوس"، فنشر عددًا من الحكايات الإن و القصص تحت عنوان "الديكاميرون السوداء"، وهو عنوانٌ مستوحى من الكتاب الإيطالي "الديكاميرون" أو "الليالي العشر"، ثم جاء زميله "ماينهوف" بكتاب "حكايات خيالية

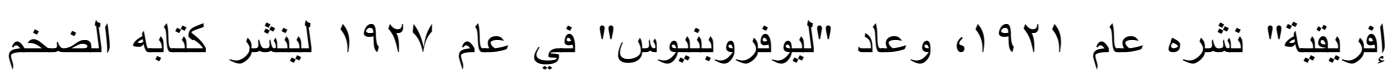

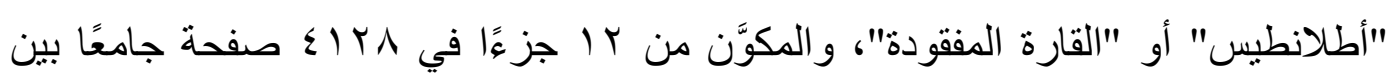
ضفافه مئات القصص و الأساطير و الخر افات الثعبية. وتعتبر محاو لات "سيدل" و "ماينهوف" و "فروبنيوس" هي التي لفتت الدارسين إلى ولى

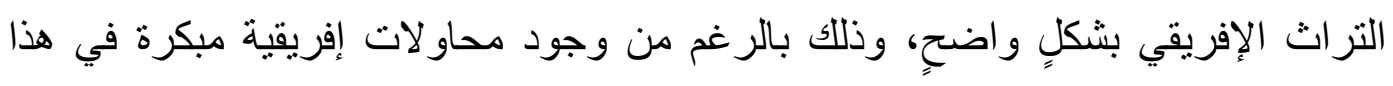

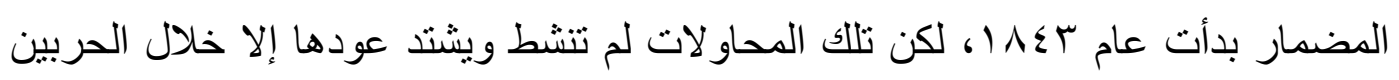
العالميتين، ثم ما تلاها من ثور ات الحرية و الاستقلال.

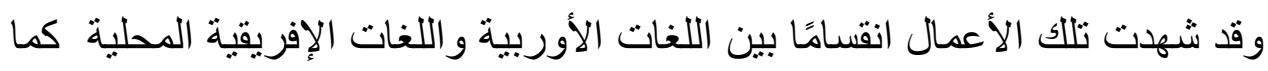

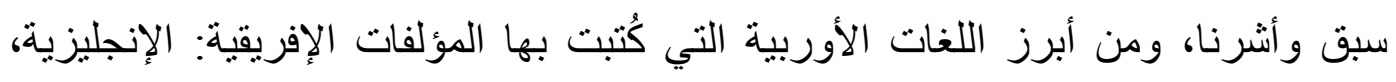

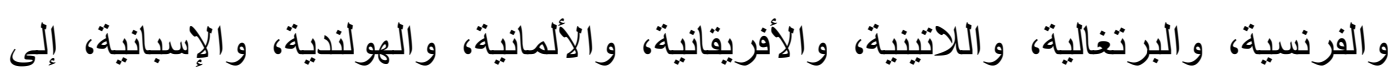


جانب اللغات الإفريقية التي وصلت إلى . . لغة، منها اء ذات أبجدية مكتوبة، ومن أهمها: السوتو، و الزوسا، و الزولو، ونيانجا، وبيمبا، وتسونجا، و الترنسفال، و غير ها الكثير. لم ينتهِ الحكي عن الأدب الإفريقي قطعًا باستدعاء أصوله، وتلمس فروعه، فدروب الأدب الإفريقي تشعبت وبدأت تزداد تعقيدًا بتدفق النور إلى إفريقيا وانتقال الحداثة إليها، منذ تحررها بدايةً من منتصف القرن الماضي، إلى وضع بعض الدول فيها الأقدام على طريق التنمية و الحضارة، ولكن أهم ما يعنينا هى المشكلات التي يتعرض لها ذلك الأدب من تشويه جرّاء كتابته بلغة المستعمر، كذلك ما بعوق نقل تلك الأعمال الأدبية وتداولها بين أطفال القارة الإفريقية لتكون كيانًا أدبيًّا وثقافيَّا موحدًا.

\section{خاتمة:}

إن إفريقيا تعيش معضلة كبيرة نظرًا لما تطمح إليه، وما تجده في الواقع العملي، ذلك

أنها كلما خططت لإجراء إصلاحٍ أدبي وثربوي داعٍ إلى تطوير لغات أصلية، اصطدمت بتنمية لغات غربية مقابل إعفاء الديون أو التخفيف منها، أي أنها تعيش بين المدق و السندان، ويتضح ذلك مثلًا في سياسة فرنسا التي تنز عم الفرنكوفونية، و أتباعها من بلجيكا وكندا، في إطار التسويق للغة الفرنسية عالميَّا، حيث تستعمل الضغط الاقتصادي على مجموعة من الدول الإفريقية الفقيرة، كما اتضح ذلك في الإعلان المثير للرئيس "ميتر ان" في قمة داكار

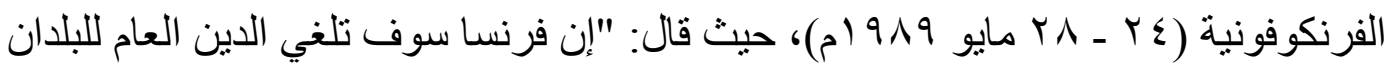
الإفريقية الخمسة والثلاثين الأفقر، وهو 17 بليون فرنلك في جملته، وفي المقابل بُنتظر من هذه البلدان أن تستمر في ضمان دور التفوق للغة الفرنسية في الحكومة". إنه من الصعوبة الخروج من هذه المآزق ما دام هناك ارتباطٌٌ اقتصادي بالدول الكبرى التي تضغط بكل ما أوتيت من قوة على الدول الفقيرة، فتوقعها فريسة؛ لتخضعها لمصالحها الخاصة، وتملي عليها ما تريد، ولا يمنعنا ذلك من أن نطر ح إمكانية تطور اللغات الإفريقية في بحوثٍ أخرى. 


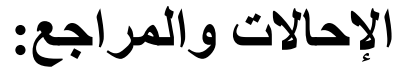

URL

http://online.wsj.com/article/SB

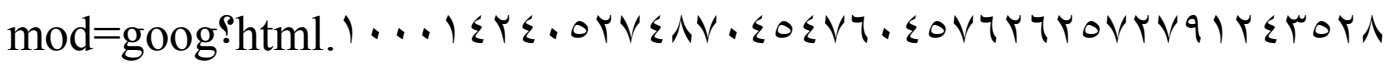

lenews_wsj

- $\quad$ languages-click-http://www.theroot.com/views/what :URL

origins, -our-about-tell-africa phons Kanano , Écolinguistique, Jean B. Ntakirutimana \& Al .. $\leqslant \mathrm{N} \bullet ، \leqslant$ rmultilinguisme et langues maternelles en Afrique, $\mathrm{p}$ :

ACALAN, Mali ، r.. $\leqslant$ Heine \& Derek Nurse Bernd, Les langues Africaines .${ }^{9}$ Karthala, Paris, $p$ de linguistiques, Déclaration universelle de droits ، $99 \wedge$ Comité :.Barcelona 6 l.p

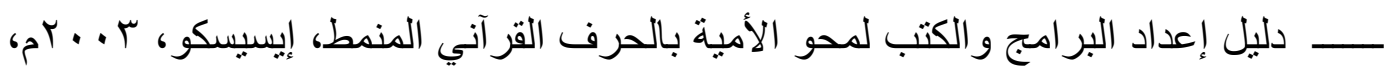

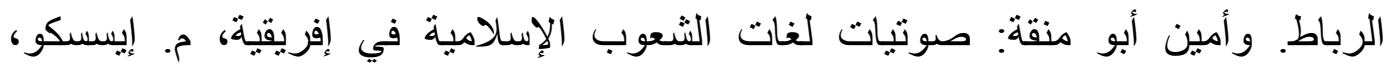

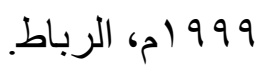

ـــــالثقافة والتتمية، الأعمال و الإنجاز ات، ص ^، جماعة من الباحثين، بالتعاون مع برتش التش

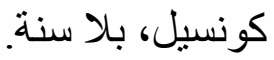
Montray KREYOL, Langues Nationales et développement en ... Aavril $r$ sjeudi 'Afrique auranne Provenzano, La langue française sauvée par إبع: 'Afrique

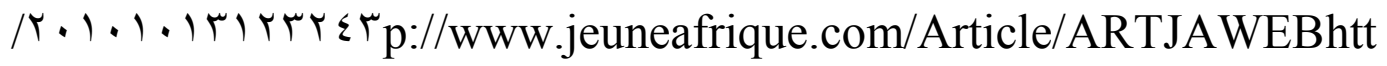
-1-par-sauvee-francaise-langue-oifla-developpement-afrique-enquete $\cdot \cdot h: 1$ rà $r \cdot 1 \cdot / 1 \cdot / /$ rafrique.html, 
Jean B. Ntakirutimana \& Alphons Kanano, Écolinguistique, ، Y es en Afrique, $p$ multilinguisme et langues maternell

$$
\begin{aligned}
& \text {.ACALAN, Mali } 6 . . \leqslant \mathrm{N} \bullet
\end{aligned}
$$

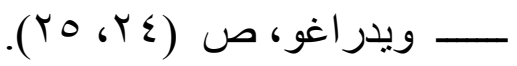

Dynamique de langue et politique $r .1 /$ Tomas BLECKE linguistique, Conférence Nationale sur le projet de document de - politique linguistique au Mali, $\mathrm{p}$

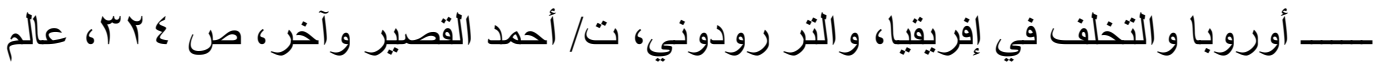
المعرفة، ع ب ا 1، 91 (ام، ويمكن مر اجعة الصفحات التي بعدها؛ فهي في غاية الأهمية.

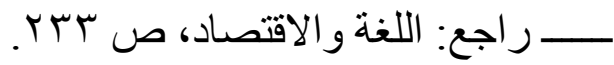
ــــ راجع: OUEDRAOGO، ص צז.

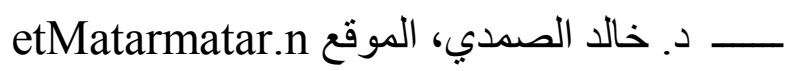
Rakissouiligri Mathieu OUEDRAOGO, planification et politiques $r$ Tlinguistiques dans certains pays sélectionnés d'afrique de 1 'ouest, $p$ r. . IEd, Mulualem Seyoum, L’UNESCO, ، $₫ \& \&$

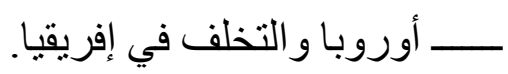

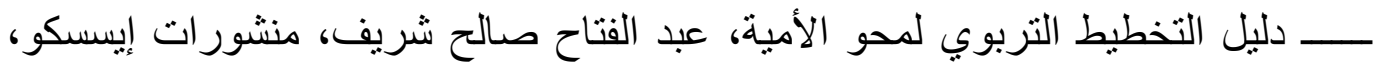

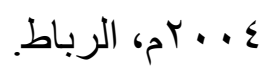
/http://arz.wikipedia.org/wiki

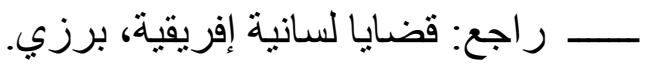

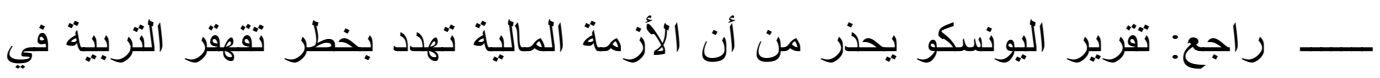

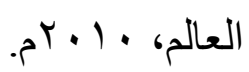

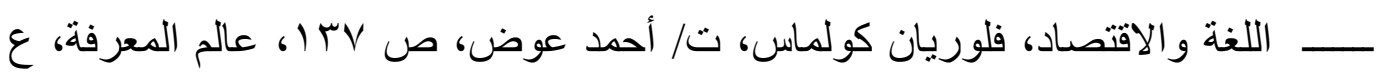

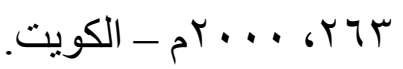

